PROCEEDINGS OF THE

AMERICAN MATHEMATICAL SOCIETY

Volume 125, Number 11, November 1997, Pages 3397-3401

S 0002-9939(97)03959-2

\title{
ON CONVEX CLASS OF PAIRS OF CONVEX BODIES
}

\author{
JERZY GRZYBOWSKI AND RYSZARD URBAŃSKI
}

(Communicated by Palle E. T. Jorgensen)

\begin{abstract}
In this paper we introduce a quotient class of pairs of convex bodies in which every member have convex union.
\end{abstract}

The space of pairs of convex bodies has been investigated in a number of papers $[3],[8],[9]$, and [12]. This space has found an application in quasidifferential calculus (cf. [1], [5], [7], [10]). A quasidifferential is represented as a pair of convex bodies and it is essential to find the minimal representation of this pair. The notion of minimal pairs was introduced in [5] and investigated in [2], [6], [7] and [11]. Some criteria of minimality are given in [6]. In this paper we investigate pairs of convex bodies with convex union. We introduced a quotient class of pairs of convex compact sets in which every member has convex union. Moreover some criteria for the convex class are given.

In this paper $X=(X, \tau)$ stands for a real locally convex vector space, and $X^{*}$ denotes the dual space of $X$. Denote by $\mathcal{K}(X)$ the family of all convex bodies in $X$, i.e., of all nonempty compact convex subsets of $X$. If $A, B$ are nonempty subsets of $X$, then $A+B$ is the usual algebraic Minkowski sum of $A$ and $B$. It may be showed that $\mathcal{K}(X)$ satisfies the order cancellation law; i.e. for every $A, B, C \in \mathcal{K}(X)$ the inclusion $A+B \subset B+C$ implies $A \subset C$ (cf. [12]). Hence it follows that $\mathcal{K}(X)$ endowed with the Minkowski sum is a commutative semigroup satisfying the law of cancellation.

Now let $\mathcal{K}^{2}(X)=\mathcal{K}(X) \times \mathcal{K}(X)$; the equivalence relation between pairs of convex bodies is given by: $(A, B) \sim(C, D)$ if and only if $A+D=B+C$. For $A, B \in \mathcal{K}(X)$ we will use the notation $A \vee B:=\operatorname{conv}(A \cup B)$, where the operation "conv" denotes the convex hull. If $A, B, C \in \mathcal{K}(X)$, and $b \in X$, then $A \vee B+C=(A \vee B)+C$ and $A+b=A+\{b\}$. We have $[a, b]=\{a\} \vee\{b\}$.

Let $f \in X^{*}, A \in \mathcal{K}(X)$ and $c \in \mathbb{R}$. We denote by $p_{A}(f):=\max _{x \in A} f(x)$ the support function of the set A. Moreover, $H_{f}^{c}:=\{x \in X \mid f(x)=c\}$ and $H_{f} A:=\left\{x \in A \mid f(x)=p_{A}(f)\right\}$, where $H_{f}^{c}$ is the hyperplane generated by the functional $f$ and the number $c$, and $H_{f} A$ is the face of $A$ with respect to $f$. For the sum of the faces of two convex bodies $A, B \subset X$ with respect to $f \in X^{*}$ the identity $H_{f}(A+B)=H_{f}+H_{f} B$ holds true. For $A \subset X$ we denote by $\partial A$ the boundary $\bar{A} \backslash A^{o}$ of the set $A$, where $\bar{A}:=\operatorname{cl} A$ and $A^{o}:=\operatorname{int} A$.

Received by the editors June 12, 1996.

1991 Mathematics Subject Classification. Primary 52A07, 90C30, 26A27.

Key words and phrases. Convex Analysis, pairs of convex sets, quasidifferential.

(C)1997 American Mathematical Society 
Let $A, B \in \mathcal{K}(X)$. The class $[A, B]:=\left\{(C, D) \in \mathcal{K}^{2}(X) \mid(A, B) \sim(C, D)\right\}$ is called convex if for every member $(C, D) \in[A, B]$ the set $C \cup D$ is convex.

Proposition 1. If $\operatorname{dim} X>1$, and $A, B \in \mathcal{K}(X)$, then $A \cup B$ is convex if and only if $\partial(A \vee B) \subset A \cup B$.

Proof. Necessity. For arbitrary sets $A, B \subset X$ we have $\partial(A \cup B) \subset \partial A \cup \partial B \subset A \cup B$. But $A \vee B=A \cup B$. Hence $\partial(A \vee B) \subset A \cup B$.

Sufficiency. Let $\operatorname{dim} X<\infty$. Given any $x \in A \vee B$ with $x \notin A$, there exist $f \in X^{*}$ and $c \in \mathbb{R}$ such that the hyperplane $H_{f}^{c}$ separates the set $\{x\}$ and $A$. We can assume that $x \in H_{f}^{c}$ and $H_{f}^{c} \cap A=\emptyset$. Take any line $l \subset H_{f}^{c}$ passing through the point $x$. Then $l \cap(A \vee B)=[p, q]$ for some $p, q \in \partial(A \vee B)$. But $\partial(A \vee B) \subset A \cup B, H_{f}^{c} \cap A=\emptyset$. Hence $p, q \in B$ and we get $x \in[p, q] \subset B$.

Now, let $\operatorname{dim} X=\infty$. Then $\partial(A \vee B)=A \vee B$, and $\partial(A \vee B) \subset A \cup B$ implies $A \vee B \subset A \cup B$. Hence $A \vee B=A \cup B$.

If $\operatorname{dim} X=1$, then for $A:=\{0\}, B:=\{1\}$ we have $\partial(A \vee B)=\{0,1\}=A \cup B$ but $A \cup B$ is not convex.

In [4] the following is proved:

Lemma. If $X$ is finite-dimensional and $A \subset X$ is a convex set, then at any point $x$ of the boundary $\partial A$ of $A$ there is a supporting hyperplane for $A$.

In the infinite-dimensional case, the above lemma does not hold true. For example let $X=l^{2}$ and we consider the Hilbert cube

$$
A:=\left\{x=\left(\xi_{n}\right) \mid \xi_{n} \in \mathbb{R}, \text { and }\left|\xi_{n}\right| \leq \frac{1}{n}\right\} .
$$

The set $A$ is compact and convex. It is easy to observe that $p_{A}(f)>0$ for every nontrivial $f \in X^{*}$. Since $A$ is compact $\partial A=A$. Moreover, $f(0)=0$ for any $f \in X^{*}$. So, there is no supporting hyperplane at 0 .

Proposition 2. If $1<\operatorname{dim} X<\infty, A, B \in \mathcal{K}(X)$, then $A \cup B$ is convex if and only if $H_{f}(A \vee B) \subset H_{f} A \cup H_{f} B$ for every $f \in X^{\star} \backslash\{0\}$.

Proof. Necessity. Given any $f \in X^{*} \backslash\{0\}$, we have

$$
p_{A \vee B}(f)=\max \left\{p_{A}(f), p_{B}(f)\right\} \text {. }
$$

Now let $p_{A}(f)<p_{B}(f)$. Then $H_{f}(A \vee B)=H_{f} B$. Analogously, if $p_{A}(f)>p_{B}(f)$, we obtain $H_{f}(A \vee B)=H_{f} A$. Suppose $p_{A}(f)=p_{B}(f)$. If $x \in H_{f}(A \vee B) \subset A \cup B$, then $x \in A$ or $x \in B$. If $x \in A$, then $x \in H_{f} A$. It follows from the above that $H_{f}(A \vee B) \subset H_{f} A \cup H_{f} B$ for every $f \in X^{*} \backslash\{0\}$.

Sufficiency. Let $x \in \partial(A \vee B)$. Since $\operatorname{dim} X<\infty$, so from the lemma it follows that $x \in H_{f}(A \vee B)$ for some nontrivial $f \in X^{*}$. And we obtain from assumption $x \in A \cup B$. Hence $\partial(A \vee B) \subset A \cup B$. Now, it follows from Proposition 1 that $A \cup B$ is convex.

Theorem 1. Let $1<\operatorname{dim} X<\infty, A, B \in \mathcal{K}(X)$. If $H_{f}(A \vee B)=H_{f} A$ or $H_{f} B$ for every $f \in X^{*} \backslash\{0\}$ then the class $[A, B]$ is convex.

Proof. We observe that

$$
H_{f}(A \vee B) \subset H_{f} A \cup H_{f} B \text { for every nontrivial } f \in X^{*} .
$$

Hence from Proposition 2 we have that $A \cup B$ is convex. 
Now, given any pair $(C, D) \in \mathcal{K}^{2}(X)$ equivalent to $(A, B)$, we have

$$
A+C \vee D=(A+C) \vee(A+D)=(A+C) \vee(B+C)=C+A \vee B .
$$

Analogously

$$
B+C \vee D=D+A \vee B
$$

We also have

$$
\begin{gathered}
H_{f} A+H_{f}(C \vee D)=H_{f} C+H_{f}(A \vee B), \\
H_{f} B+H_{f}(C \vee D)=H_{f} D+H_{f}(A \vee B) \text { for every } f \in X^{*} \backslash\{0\} .
\end{gathered}
$$

But

$$
H_{f}(A \vee B)=H_{f} A \text { or } H_{f} B \text {. }
$$

Hence from the law of cancellation we have

$$
H_{f}(C \vee D)=H_{f} C \text { or } H_{f} D \text { for every } f \in X^{*} \backslash\{0\} .
$$

This implies that

$$
H_{f}(C \vee D) \subset H_{f} C \cup H_{f} D \text { for every } f \in X^{*} \backslash\{0\} .
$$

Hence we obtain from Proposition 2 that $C \cup D$ is convex.

The condition $H_{f}(A \vee B) \subset H_{f} A \cup H_{f} B$ in Theorem 1 is not sufficient. For example, let $A, B \in \mathcal{K}\left(\mathbb{R}^{2}\right)$,

$$
A:=\{(x, y) \mid 0 \leq x \leq 1,0 \leq y \leq 1\}, B:=\{(1,0)\}+A .
$$

Then $A \vee B=\{(x, y) \mid 0 \leq x \leq 2,0 \leq y \leq 1\}, H_{f}(A \vee B) \subset H_{f} A \cup H_{f} B$ for $f \in X^{*} \backslash\{0\}$. Define $C:=\{(0,0)\}, D:=\{(1,0)\}$. Then $A+D=B+C$, but $C \cup D=\{(0,0),(1,0)\}$ is not convex.

Example 1. Convex classes

i) Take any $(A, B) \in \mathcal{K}^{2}(X)$ such that $A \subset B$ and any $(C, D) \in \mathcal{K}^{2}(X)$ being an equivalent pair to $(A, B)$. Then $B+C=A+D \subset B+D$ and from the order law of cancellation, we have $C \subset D$. Hence $C \cup D=D$. It is obvious that $\partial(A \vee B) \subset A \cup B$.

ii) Let $X=\mathbb{R}^{2}$ and $R>0$. Consider the closed ball $\mathbb{B}((0,0), R)$ and let

$$
a:=\left(-\frac{1}{2} \cdot \sqrt{2} \cdot R, \frac{1}{2} \cdot \sqrt{2} \cdot R\right), \quad b:=\left(\frac{1}{2} \cdot \sqrt{2} \cdot R, \frac{1}{2} \cdot \sqrt{2} \cdot R\right) .
$$

Define

$$
\begin{aligned}
& A:=\left\{(x, y) \in \mathbb{B}((0,0), R) \mid-\frac{1}{2} \cdot \sqrt{2} \cdot R \leq x \leq \frac{1}{2} \cdot \sqrt{2} \cdot R\right\}, \\
& B:=\left\{(x, y) \in \mathbb{B}((0,0), R) \mid-\frac{1}{2} \cdot \sqrt{2} \cdot R \leq y \leq \frac{1}{2} \cdot \sqrt{2} \cdot R\right\}
\end{aligned}
$$

(see Figure 1).

We have $A \cup B=\mathbb{B}((0,0), R)$ and $A \cap B=-a \vee(-b) \vee a \vee b$. Since $H_{f}(A \vee B)=$ $H_{f} A$ or $H_{f} B$ for every nonzero $f \in X^{*}$, it follows from the above theorem that the class $[A, B]$ is convex.

Theorem 2. Let $X=\mathbb{R}^{2}, A, B \in \mathcal{K}(X)$. Then the class $[A, B]$ is convex if and only if $H_{f}(A \vee B)=H_{f} A$ or $H_{f} B$ for all $f \in X^{*} \backslash\{0\}$. 




FiguRE 1

Proof. Necessity. Assume that $H_{f} A \neq H_{f}(A \cup B) \neq H_{f} B$ for some $f \in X^{*} \backslash\{0\}$. It follows from the assumption that $p_{A}(f)=p_{B}(f)$ and the faces $H_{f} A$ and $H_{f} B$ are parallel segments and not one-point sets. In fact, $H_{f} A$ and $H_{f} B$ are contained in one line. Denote $H_{f} A:=a \vee b, H_{f} B:=c \vee d$, where $a, b, c, d \in \mathbb{R}^{2}$ and assume that $d-c=k \cdot(b-c)$ for some $k \geq 1$. Let $e \in \mathbb{R}^{2}$ be a point $H_{f} T=e$ where $I=a \vee b$ and $T=I \vee e$. Then $H_{-f} T=I$. Denote $J:=(c-a) \vee(d-b)$.

We have

$$
\begin{gathered}
H_{f}(A+T)=I+e, \quad H_{f}(B+T)=I+J+e, \\
H_{-f}(A+T)=I+H_{-f} A, \quad H_{-f}(B+T)=I+H_{-f} B .
\end{gathered}
$$

Therefore, the segment $I$ is a summand of both $A+T$ and $B+T$. Let $A^{\prime}, B^{\prime} \in$ $\mathcal{K}(X), A+T=A^{\prime}+I$ and $B+T=B^{\prime}+I$.

We have

$$
\begin{gathered}
H_{f} A^{\prime}+I=H_{f} A^{\prime}+H_{f} I=H_{f}(A+T)=I+e, \\
H_{f} B^{\prime}+I=I+J+e .
\end{gathered}
$$

It follows from these equations that $H_{f} A^{\prime}=e$ and $H_{f} B^{\prime}=J+e$. Since $H_{f} B$ does not contain $H_{f} A$ then $0 \notin J$, and $e \notin J+e$. Therefore, $H_{f} A^{\prime} \cap H_{f} B^{\prime}=\emptyset$. Since $p_{A^{\prime}}(f)=p_{B^{\prime}}(f)$ then $H_{f}\left(A^{\prime} \vee B^{\prime}\right)=H_{f} A^{\prime} \vee H_{f} B^{\prime} \neq H_{f} A^{\prime} \cup H_{f} B^{\prime}=H_{f}\left(A^{\prime} \cup B^{\prime}\right)$. According to Proposition 2, the pair $\left(A^{\prime}, B^{\prime}\right)$ is not convex while $\left(A^{\prime}, B^{\prime}\right) \in[A, B]$. This contradicts the assumption of our theorem.

Sufficiency. It follows immediately from Theorem 1.

Example 2. Let $X:=\mathbb{R}^{3}, A:=\{(x, y, z) \in \mathbb{B}((0,0), R) \mid x \leq 0, z \leq 0\}, B:=$ $\{(x, y, z) \in \mathbb{B}((0,0), R) \mid x \geq 0, z \leq 0\}$ (see Figure 2). Denote $f(x, y, z):=z$.

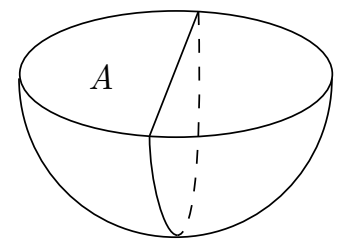

FiguRE 2 
The functional $f \in X^{*} \backslash\{0\}$ and $A^{\prime}:=H_{f} A, B^{\prime}:=H_{f} B \subset Y:=\mathbb{R}^{2} \times\{0\}$. Notice that $A^{\prime}$ and $B^{\prime}$ are half-discs and $A^{\prime} \cup B^{\prime}$ is a disc. Therefore for all $F^{\prime} \in$ $Y^{*} \backslash\{0\}, H_{f^{\prime}}\left(A^{\prime} \vee B^{\prime}\right)=H_{f^{\prime}} A^{\prime}$ or $H_{f^{\prime}} B^{\prime}$. According to Theorem 1 , the class $\left[A^{\prime}, B^{\prime}\right] \in \mathcal{K}^{2}(Y) / \sim$ is convex. Therefore, for any pair $(C, D) \in[A, B]$. the pair $\left(H_{f} C, H_{f} D\right)$ is convex. Then $H_{f}(C \vee D) \subset H_{f} C \cup H_{f} D$. Now, if $g \in X^{*}, g \neq k f$, where $k \geq 0$ then $H_{g}(A \vee B)$ is one-point set equal to $H_{g} A$ or $H_{g} B$. Therefore, $H_{g}(C \vee D)$ must be equal to $H_{g} C$ or $H_{g} D$. Now, $H_{g}(C \vee D) \subset H_{g} C \cup H_{g} D$ and, according to Proposition 2, $H_{f} A \neq H_{f}(A \vee B) \neq H_{f} B$. Therefore, we may not replace the space $X=\mathbb{R}^{2}$ in Theorem 2 any other more-than-two dimensional space.

\section{REFERENCES}

1. V. F. Demyanov, A. M. Rubinov, Quasidifferential Calculus, Optimization Software Inc., Publication Division, New York 1986.

2. J. Grzybowski, Minimal pairs of convex compact sets, Arch. Math. Vol. 63 (1994), 173-181. MR 95h:52001

3. L. Hörmander, Sur la fonction d'appui des ensembles convex dans une espace localement convexe, Arkiv for Math. 3 (1954), 181-186. MR 16:831e

4. von K. Leichtweiß, Konvexe Mengen, VEB Deutscher Verlag der Wissenschaften, Berlin 1980. MR 81b:52001

5. D. Pallaschke, S. Scholtes and R. Urbański, On minimal pairs of compact convex sets, Bull. Polish Acad. Sci. Math. 39 (1991), 1-5. MR 93j:52002

6. D. Pallaschke and R. Urbański, Some criteria for the minimality of pairs of compact convex sets, Zeitschrift für Opertations Research 37 (1993), 129-150. MR 94e:49004

7. D. Pallaschke, R. Urbański, Reduction of quasidifferentials and minimal representations, Math. Programming 66 (1994), 161-180. MR 95i:49030

8. A. G. Pinsker, The space of convex sets of a locally convex space, Trudy Leningrad Engineering-Economics Institute, 63 (1966), 13-17. MR 36:5653

9. H. Rådström, An embedding theorem for spaces of convex sets, Proc. Amer. Math. Soc. 3 (1952), 165-169. MR 13:659c

10. A. M. Rubinov, I. S. Akhundov, Differences of compact sets in the sense of Demyanov and its application to non-smooth-analysis, Optimization 23 (1992), 179-189. MR 94j:49023

11. S. Scholtes, Minimal pairs of convex bodies in two dimensions, Mathematika 39 (1992), 267-273. MR 93m:52012

12. R. Urbański, A generalization of the Minkowski-Rådström-Hörmander Theorem, Bull. Polish Acad. Sci. Math. 24 (1976), 709-715. MR 56:1027

Faculty of Mathematics, and Computer Science, Adam Mickiewicz University, MateJKI 48/49, 60-769 Poznań, Poland

E-mail address: jgrz@math.amu.edu.pl

E-mail address: rich@math.amu.edu.pl 